\title{
Serological testing for SARS-CoV-2 in Bosnia and Herzegovina: first report
}

Adna Ašić ${ }^{1}$, Jasminka Prguda-Mujić², Lana Salihefendić1,3, Larisa Bešić ${ }^{1}$, Daria Ler ${ }^{2}$, Ivana Čeko Almira Hadžović-Džuvo ${ }^{4,5}$, Sead Jažić ${ }^{6}$, Rijad Konjhodžić ${ }^{3}$, Damir Marjanovićc ${ }^{1,7}$

${ }^{1}$ Department of Genetics and Bioengineering, International Burch University, Sarajevo, Bosnia and Herzegovina

2Eurofarm Molecular Diagnostics Laboratory, Eurofarm Centre, Sarajevo,

Bosnia and Herzegovina

${ }^{3}$ Alea Genetic Centre, Health Institute Alea Dr. Kandić, Sarajevo,

Bosnia and Herzegovina

${ }^{4}$ Department of Human Physiology, University of Sarajevo, Sarajevo,

Bosnia and Herzegovina

${ }^{5}$ Polyclinic and Daily Hospital Dr. Al-Tawil, Sarajevo, Bosnia and Herzegovina

${ }^{6}$ Department of Microbiology Diagnostics, General Hospital Dr. Abdulah Nakaš,

Sarajevo, Bosnia and Herzegovina

IInstitute for Anthropological Research, University of Zagreb, Zagreb, Croatia

Submitted: 13 November 2020; Accepted: 8 March 2021;

Online publication: 18 March 2021

Arch Med Sci 2021; 17 (3): 823-826

DOI: https://doi.org/10.5114/aoms/134143

Copyright @ 2021 Termedia \& Banach

\section{Abstract}

Introduction: Serological detection of SARS-CoV-2-specific immunoglobulins G (IgG) and $M(\operatorname{IgM})$ antibodies is becoming increasingly important in the management of the COVID-19 pandemic.

Methods: We report the first results of COVID-19 serological testing in Bosnia and Herzegovina on 2841 samples collected and analysed in 2 medical institutions in Sarajevo. Antibody detection was performed using commercially available kits.

Results: In the first cohort, 43 IgM-positive/IgG-negative and 16 IgM-positive/lgG-positive individuals were detected, corresponding to $3.41 \%$ of participants having developed antibodies. In the second cohort, $4.28 \%$ participants were found to be IgM-negative/lgG-positive.

Conclusions: Our results suggest the need for population-wide serological surveying in Bosnia and Herzegovina.

Key words: COVID-19, immunoglobulins G, immunoglobulins M, molecular testing, serological testing.

Severe acute respiratory syndrome coronavirus 2 (SARS-CoV-2) is causing a novel COVID-19 infection [1, 2]. While the standard diagnostic test is molecular analysis using qRT-PCR, serological testing for COVID-19 is becoming increasingly important in research community and immunity surveillance efforts. Serological testing is performed on whole blood, serum, or plasma samples in a relatively simple and rapid procedure, requiring less expertise and simpler laboratory settings compared to molecular methods. Tests are designed to detect either total immunoglobulins (Ig) or to differentiate between immunoglobulins $G(\operatorname{lgG})$ and immunoglobulins $M(\operatorname{IgM})$ fractions [3, 4]. Previous studies have reported sensitivity and specificity of $77.3 \%$ and $100 \%$ for IgM, respectively, and $83.3 \%$ and

\author{
Corresponding author: \\ Adna Ašić \\ Department of Genetics \\ and Bioengineering \\ International Burch \\ University \\ Francuske revolucije bb \\ Sarajevo 71000 \\ Bosnia and Herzegovina \\ E-mail: adna.asic@ibu.edu.ba
}


95\% for IgG, respectively [5]. Serological assays directed towards SARS-CoV-2 are based on detection of antibodies against a 2-subunit viral spike (S) protein, with $\mathrm{S} 1$ being responsible for receptor binding and $\mathrm{S} 2$ for fusion $[6,7]$.

The aim of this preliminary study is to offer the first report on serological testing for SARS-CoV-2 in Bosnia and Herzegovina (B\&H).

Methods. Sample collection and analysis in the first cohort. This study was conducted in accordance with the Helsinki Declaration for research involving human subjects. All patient samples were collected during routine laboratory operations and anonymized prior to manuscript preparation.

A total of 1720 whole blood and/or pharyngeal swab samples were collected and tested for IgM/ IgG antibodies, as well as for viral RNA at the Dr. Abdulah Nakaš General Hospital in Sarajevo, B\&H in April and May 2020. All samples were collected as a part of the hospital triage procedure. Blood samples were collected in a tube containing an anticoagulant and were tested immediately. Additionally, 131 nasopharyngeal and oropharyngeal swab samples were taken from a subset of the study population for qRT-PCR testing, according to patients' clinical profiles and based on the recommendation from the attending medical doctor. For serological testing, the Artron One-Step Novel Coronavirus (COVID-19) IgM/lgG Antibody Test Kit (Artron Laboratories, Burnaby, BC, Canada) was used. For molecular testing, RNA was extracted using QIAamp Viral RNA Mini Kit (Qiagen, Hilden, Germany), followed by RNA quantification using a Qubit fluorometer (Thermo Fisher Scientific, Waltham, MA, USA). Two thermal cyclers were used for viral RNA amplification, namely QuantStudio $^{\text {TM }} 7$ Flex Real-Time PCR System and QuantStudio 5 Dx Real-Time PCR (qPCR) System (Thermo Fisher Scientific, Waltham, MA, USA). Diagnovital ${ }^{\circledR}$ 2019-nCoV Real-Time PCR Kit (RTA Laboratories, Gebze/Kocaeli, Turkey) was used in

Table I. Clinical and demographic characteristics of serologically positive patients from Sarajevo, B\&H

\begin{tabular}{|lc|}
\hline Total serologically positive & 107 \\
\hline Female & 50 \\
\hline Male & $54^{\star}$ \\
\hline Sore throat & 0 \\
\hline Cough & 4 \\
\hline Fever & 5 \\
\hline Muscle pain & 1 \\
\hline Short breath & 4 \\
\hline Traveling & 2 \\
\hline Contact with risk group & 52 \\
\hline \multirow{*}{*}{ There were cases where sex was unknown. }
\end{tabular}

amplification reactions. Internal control was HEC (human extraction control), which was amplified with every sample. In addition, each run had manufacturer-provided positive and negative controls, as well as an extraction negative control, which was treated as a sample.

Sample collection and analysis in the second cohort. A total of 1121 individuals were tested for COVID-19-specific IgG and IgM antibodies at the Eurofarm Centre Laboratory in Sarajevo between 21 April 2020 and 17 July 2020. Venous blood samples were collected in a plain tube without anticoagulant. All collected serum samples were immediately processed with the Dynamiker Biotechnology Co. rapid test (Tianjin, China) to detect SARS-CoV-2 IgG/IgM antibodies. The test was internally validated in the laboratory on 5 PCR-positive patients (day 21 after detection) and 5 healthy PCR-negative volunteers.

Statistical analysis. All analyses were performed using Excel 2010 (Microsoft Corporation, Redmond, USA). The exact test of goodness-of-fit was used to determine the effect of age on the frequency of COVID-19-positive individuals, in addition to comparing the frequency of individuals who were most probably still actively infected (IgM-positive, IgG-negative) and individuals at a later stage of the infection (IgM-positive, IgG-positive). Furthermore, we determined the effect of a reported underlying condition and sex on the frequency of IgG-positive cases. The 2-sample $t$-test was used to compare the mean age among female and male individuals.

Results. A total of 59 (3.41\%) out of 1720 individuals were confirmed to be positive for COVID-19 antibodies in the first cohort. The mean age of individuals who tested positive was 61.46 years. The number of individuals who were older than 50 years $(86.44 \%)$ was significantly higher than the number of individuals who were younger than 50 years $\left(13.56 \%, p=9.05 \times 10^{-9}\right)$. In addition, the mean age of female participants (66.57 years), who comprised $50 \%$ of the individuals who tested positive for COVID-19, was found to be significantly higher than the mean age of male participants $\left(56.37, p=8.5 \times 10^{-3}\right)$. None of the reported symptoms were shown to have been in correlation with either sex or age of the participants (data not shown) (Table I).

Out of 59 antibody-positive individuals, $43(72.88 \%)$ tested positive for IgM but negative for $\mathrm{lgG}$, indicating that these individuals were most probably still actively infected $[5,8]$. The remaining $16(27.11 \%)$ individuals tested positive for both antibodies, indicating either a later stage of active infection or convalescence $[5,8]$ (Table II). The number of individuals that were IgM-positive, IgG-negative was significantly higher 
than the number of individuals that were IgM-positive and lgG-positive $\left(p=5.84 \times 10^{-4}\right)$.

When it comes to RT-qPCR testing, out of 131 swab samples, 3 tested positive for the presence of viral RNA. One PCR-positive individual tested IgM-positive/lgG-negative, while 2 PCR-positive individuals tested IgM-negative/lgG-negative. Furthermore, 128 samples tested PCR-negative, including 106 samples that were IgM-negative/ IgG-negative, 11 samples that were IgM-positive/ IgG-negative, and 11 samples that were IgM-positive/lgG-positive in serological testing (Table III).

In the second cohort, 48 (4.28\%) of 1121 individuals tested positive for the IgG antibody, while none of the individuals tested positive for the IgM antibody (Table II), indicating either convalescence or previous infection or exposure [5, 8]. The mean age of the IgG-positive individuals was 43.38 years, with the youngest IgG-positive participant being 18 and the oldest 68 years old. The mean age of all participants from this cohort was 41.51 years, ranging from 1 to 88 years. Out of 48 individuals, 22 (45.83\%) were female and $26(54.17 \%)$ were male, indicating no significant difference in IgG incidence between sexes ( $p=$ $0.665)$. The mean age of female and male participants was 43.2 and 45.5 years, respectively, with no statistically significant difference in mean age between female and male groups $(p=0.22)$. Five of the IgG-positive individuals were also previously confirmed to be positive for COVID-19 via PCR testing $(0.45 \%$ out of all tested individuals). None of the individuals who tested positive for the IgG antibody showed associated symptoms (Table I).

Discussion. Serological tests may play an important role in determining the overall prevalence of COVID-19 in the Bosnian-Herzegovinian population, and may also be important in determining the prevalence of asymptomatic infections.

Our early results indicate that $3.41 \%$ of study participants from Dr. Abdulah Nakaš General Hospital of Sarajevo and $4.28 \%$ of individuals tested in the Eurofarm Centre Laboratory in Sarajevo had developed SARS-CoV-2-specific antibodies. Additional molecular detection of viral RNA in the first set of samples showed that it was detected in one IgM-positive/lgG-negative sample and was not detected in 11 samples of IgM-positive/lgG-negative individuals and 11 samples that were IgM-positive/lgG-positive. On the other hand, 5 out of 48 (10.4\%) IgM-negative/lgG-positive participants from the Eurofarm Centre Laboratory set were previously confirmed to be PCR-positive through the governmental testing procedure, implying that serological testing correctly showed previous infection with SARS-CoV-2. These results further emphasize different roles of serological and molecular assays for SARS-CoV-2 antibodies
Table II. An overview of serological testing results from 2 laboratories in Sarajevo, B\&H

\begin{tabular}{|lcc|}
\hline Variable & $\begin{array}{c}\text { Dr. Abdulah } \\
\text { Nakaš General } \\
\text { Hospital }\end{array}$ & $\begin{array}{c}\text { Eurofarm Centre } \\
\text { Laboratory }\end{array}$ \\
\hline Total tested & 1720 & 1121 \\
\hline IgG+/IgM+ & 16 & 0 \\
\hline IgG-/IgM+ & 43 & 0 \\
\hline IgG+/IgM- & 0 & 48 \\
\hline IgG-/IgM- & 1661 & 1073 \\
\hline
\end{tabular}

Table III. Comparison of serological vs. molecular testing of 131 selected samples from Dr. Abdulah Nakaš General Hospital of Sarajevo

\begin{tabular}{|lcc|}
\hline Variable & PCR positive & PCR negative \\
\hline IgM-/IgG- & 2 & 106 \\
\hline $\lg M+/ \operatorname{lgG}-$ & 1 & 11 \\
\hline $\lg M+/ \operatorname{lgG}+$ & 0 & 11 \\
\hline $\lg M-/ \lg G+$ & 0 & 0 \\
\hline
\end{tabular}

and RNA, respectively, as well as the importance of time of sampling with respect to the infection start time.

The IgM/lgG antibodies for SARS-CoV-2 were found to develop 6-15 days post disease onset [9-13]. The presence of antibodies was detected in $<40 \%$ patients within 1 week of infection onset, and rapidly increased to $100 \%$ (total antibodies), $94.3 \%$ (IgM), and $79.8 \%$ (IgG) from day 15 after onset [11]. Previous studies suggested that the fraction of IgM-positive patients was decreasing as the time passing since infection confirmation [14]. Other reports found that an antibody response is displayed between days 10 and 21 after the infection onset, with evidence that the detection in some cases can take 28 days or more, while in a small number of cases IgM and/or IgG antibodies were not detected at all during the study. It is also necessary to note that the virus-specific IgG is significantly lower in asymptomatic than in symptomatic individuals in the acute phase [9]. This issue is even more pronounced considering that $40-45 \%$ of COVID-19 cases appear asymptomatic [15].

Our results represent a good approximation of population-level figures in the early (spring 2020) pandemic stage, because participants in the study were selected through regular hospital triage procedure or were tested for commercial purposes in an unbiased manner. Lower figures obtained in this study are expected, considering that the period in which this study was conducted coincides with the lockdown and restrictive measures in B\&H. Study limitations include the limited availability of commercial kits for serological testing, as well as limited financial resources, in the early 
stage of the COVID-19 pandemic. For this reason, we were unable to perform routine and repeated molecular and serological test monitoring of all participants, but the selection of patients for both types of analyses was made upon the decision of the attending medical doctor.

In conclusion, this study represents the very first results of a large-scale serological testing effort in B\&H conducted on 2841 samples in 2 laboratories in Sarajevo during early stage (spring 2020) of the COVID-19 pandemic in B\&H. The obtained results suggested a relatively small percentage of COVID-19 serologically positive individuals within the Bosnian-Herzegovinian population. We obtained $3.41 \%$ of IgM-positive individuals in the first, as well as $4.28 \%$ IgG-positive/ IgM-negative in the second, Sarajevo study group. Serological testing is expected to play an important role in future when it comes to analysis of the extent of COVID-19 epidemics in B\&H, as well as to follow the dynamics of infection and frequency of seropositive individuals. For such efforts, future optimization of existing procedures and sampling methodology will be required.

\section{Acknowledgments}

Adna Ašić, Jasminka Prguda-Mujić, Lana Salihefendić, Larisa Bešić contributed equally.

Institutes where the work was conducted: General Hospital Dr. Abdulah Nakaš, Sarajevo, Bosnia and Herzegovina; Eurofarm Centre, Sarajevo, Bosnia and Herzegovina.

\section{Conflict of interest}

The authors declare no conflict of interest.

\section{References}

1. Azeez SA, Alhashim ZG, Al Otaibi WM, et al. State-ofthe-art tools to identify druggable protein ligand of SARS-CoV-2. Arch Med Sci 2020; 16: 497-507.

2. Borgio JF, Alsuwat HS, Al Otaibi WM, et al. State-of-theart tools unveil potent drug targets amongst clinically approved drugs to inhibit helicase in SARS-CoV-2. Arch Med Sci 2020; 16: 508-18.

3. Krammer F, Simon V. Serology assays to manage COVID-19. Science 2020; 368: 1060-1.

4. Loeffelholz MJ, Tang YW. Laboratory diagnosis of emerging human coronavirus infections - the state of the art. Emerg Microbes Infect 2020; 9: 747-56.

5. Xiang F, Wang X, He X, et al. Antibody detection and dynamic characteristics in patients with COVID-19. Clin Infect Dis 2020; 71: 1930-4.

6. Krammer F, Simon V. Serology assays to manage COVID-19. Science 2020; 368: 1060-1.

7. Tang YW, Schmitz JE, Persing DH, Stratton CW. Laboratory diagnosis of COVID-19: current issues and challenges. J Clin Microbiol 2020; 58: e00512-20.

8. Stowell S, Guarner J. Role of serology in the COVID-19 pandemic [published online ahead of print, 2020 May 1]. Clin Infect Dis 2020; 71: 1935-6.
9. Long QX, Deng HJ, Chen J, et al. Antibody responses to SARS-CoV-2 in COVID-19 patients: the perspective application of serological tests in clinical practice. medRxiv2020; PPR118104.

10. Woelfel R, Corman VM, Guggemos W, et al. Clinical presentation and virological assessment of hospitalized cases of coronavirus disease 2019 in a travel-associated transmission cluster. medRxiv 2020; PPR116585.

11. Zhao J, Yuan $\mathrm{Q}$, Wang $\mathrm{H}$, et al. Antibody responses to SARS-CoV-2 in patients of novel coronavirus disease 2019. Clin Infect Dis 2020; 71: 2027-34.

12. Liu W, Liu L, Kou G, et al. Evaluation of nucleocapsid and spike protein-based enzyme-linked immunosorbent assays for detecting antibodies against SARS-CoV-2. J Clin Microbiol 2020; 58: e00461-20.

13. Wan WY, Lim SH, Seng EH. Cross-reaction of sera from COVID-19 patients with SARS-CoV assays. medRxiv 2020; 2020.03.17.20034454

14. Jin Y, Wang M, Zuo Z, et al. Diagnostic value and dynamic variance of serum antibody in coronavirus disease 2019. Int J Infect Dis 2020; 94: 49-52.

15. Oran DP, Topol EJ. Prevalence of asymptomatic SARS CoV-2 infection: a narrative review. Ann Intern Med 2020; M20-3012. 